\title{
Use of a modified Delphi process to develop research priorities in major trauma
}

\author{
Luke McElroy $^{1}(1) \cdot$ Lisa Robinson $^{2} \cdot$ Ceri Battle $^{3} \cdot$ Lynn Laidlaw $^{4} \cdot$ Alistair Teager $^{5} \cdot$ Louis de Bernard $^{6}$. \\ Jack McGillivray $^{7} \cdot$ Kevin Tsang $^{8}$ - Steve Bell ${ }^{9}$. Caroline Leech ${ }^{10} \cdot$ Max Marsden $^{11} \cdot$ Richard Carden $^{11} \cdot$ Kirsty Challen $^{12}$. \\ George Peck ${ }^{8} \cdot$ Kate Hancorn $^{13} \cdot$ Ross Davenport ${ }^{11} \cdot$ Karim Brohi $^{11} \cdot$ Michael S. J. Wilson $^{1}$ on behalf of NaTRIC ${ }^{14}$
}

Received: 18 February 2021 / Accepted: 5 June 2021 / Published online: 16 June 2021

○) Springer-Verlag GmbH Germany, part of Springer Nature 2021

\begin{abstract}
Purpose The burden of major trauma within the UK is ever increasing. There is a need to establish research priorities within the field. Delphi methodology can be used to develop consensus opinion amongst a group of stakeholders. This can be used to prioritise clinically relevant, patient-centred research questions to guide future funding allocations. The aim of our study was to identify key future research priorities pertaining to the management of major trauma in the UK.

Methods A three-phased modified Delphi process was undertaken. Phase 1 involved the submission of research questions by members of the trauma community using an online survey (Phase 1). Phases 2 and 3 involved two consecutive rounds of prioritisation after questions were subdivided into 6 subcategories: Brain Injury, Rehabilitation, Trauma in Older People, Pre-hospital, Interventional, and Miscellaneous (Phases 2 and 3). Cut-off points were agreed by consensus amongst the steering subcommittees. This established a final prioritised list of research questions.

Results In phase 1, 201 questions were submitted by 65 stakeholders. After analysis and with consensus achieved, 186 questions were taken forward for prioritisation in phase 2 with 114 included in phase 3.56 prioritised major trauma research questions across the 6 categories were identified with a clear focus on long-term patient outcomes. Research priorities across the patient pathway from roadside to rehabilitation were deemed of importance.

Conclusions Consensus within the major trauma community has identified 56 key research questions across 6 categories. Dissemination of these questions to funding bodies to allow for the development of high-quality research is now required. There is a clear indication for targeted multi-centre multi-disciplinary research in major trauma.
\end{abstract}

Keywords Major trauma $\cdot$ Trauma surgery $\cdot$ Delphi $\cdot$ Research priorities

Luke McElroy

lukem68@gmail.com

1 Department of General Surgery, Forth Valley Royal Hospital, Larbert FK5 4WR, UK

2 Rehabilitation Department, Newcastle Upon Tyne Hospitals NHS Foundation Trust, Royal Victoria Infirmary, Newcastle Upon Tyne NE1 4LP, UK

3 Physiotherapy Department, Morriston Hospital, Swansea SA6 6NL, UK

4 Edinburgh, Scotland, UK

5 Salford Royal NHS Foundation Trust, Salford M6 8HD, UK

6 Medical Sciences Division, University of Oxford, Oxford, UK

7 Carnoustie, UK
8 Division of Surgery, St Mary's Hospital, Imperial College London, Paddington, London W2 1NY, UK

9 Medical Directorate, North West Ambulance Service NHS Trust, Bolton BL1 5DD, UK

10 University Hospitals Coventry and Warwickshire NHS Trust, Clifford Bridge Road, Walsgrave, Coventry CV2 2DX, UK

11 Centre for Trauma Sciences, Blizard Institute, Queen Mary University of London, London, UK

12 Lancashire Teaching Hospitals NHS Foundation Trust, Preston PR2 9HT, UK

13 Trauma Service, Barts Health NHS Trust, The Royal London Hospital, Whitechapel, London E1 1FR, UK

14 National Trauma Research and Innovation Collaborative, London, UK 


\section{Introduction}

Major trauma is one of the most common causes of morbidity and mortality worldwide [1]. The burden of major trauma is increasing in the UK with over 20,000 cases of Major trauma annually in England [2]. There is no consensus regarding research priorities within the multi-disciplinary field of major trauma. In the UK, the National Institute for Health and Care Excellence (NICE) has identified that there is a need to establish research priorities within major trauma, making four recommendations for future major trauma research in 2016. However, these recommendations were based on the opinion of the NICE guideline group and not by consensus from the major trauma community [3].

The demographic of major trauma is evolving worldwide. In 2017, the trauma audit and research network (TARN) reported that blunt trauma in older people has become the most common cause of major trauma in the UK [4]. This is clearly a significantly different cohort to the classically described high impact penetrating trauma that has shaped trauma research worldwide in the last few decades. This changing face of trauma requires consensus driven questions to address the research priorities affecting this new demographic.

A modified Delphi process can be used to develop a list of research priorities by consensus from a group of experts in that field. The Delphi process was originally developed by the US military as a systematic, interactive forecasting method, based on the principle that forecasts or decisions

Fig. 1 Summary of prioritisation process from a structured group of stakeholders are more accurate than those from individuals or unstructured groups. It takes place over multiple rounds. As the rounds progress, the range of answers decreases, and the group converge towards the 'correct' answers. This specific approach involves utilising 3 rounds of question prioritisation by key stakeholders, to arrive at a final list of prioritised research questions within that field. This approach has been successfully utilised by many groups to improve efficiency and clinical utility of completed research [5-8]. To our knowledge, limited attempts have been made to determine research priorities in major trauma, and none by consensus of key stakeholders including multi-disciplinary team members and patient groups. The aim of our study was to utilise the National Trauma Research and Innovation Collaborative (NaTRIC) network to undertake a modified Delphi process to identify key research questions pertaining to the management of major trauma, involving key stakeholders in major trauma.

\section{Methods}

The National Trauma Research and Innovation Collaborative (NaTRIC) was established in 2018 with the primary objective to increase engagement in trauma research [9]. NaTRIC is a multiprofessional, multispecialty group of healthcare professionals and lay members. NaTRIC aims to harness the combined power of the national trauma networks to produce national, multi-centre studies. Using the NaTRIC network, a three-phased modified Delphi process was undertaken between February 2019 and July 2020

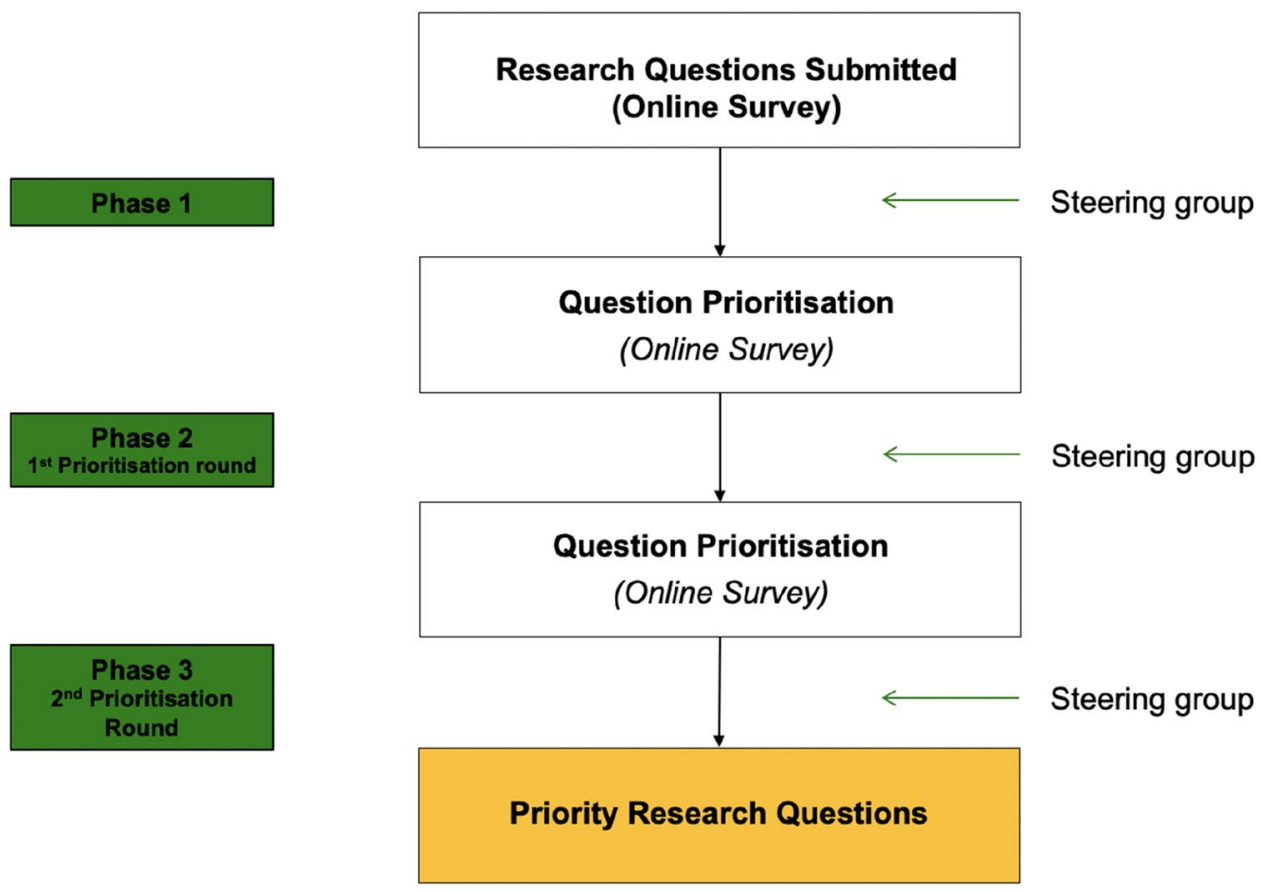


(Fig. 1). This included two distinct phases of prioritisation by expert stakeholders utilising established methodology previously described for a number of clinical projects [10-12]. Research ethical approval was not required for this study, as per the online National Research Ethics Service tool [13]. Phase 1 ran between February 8th 2019 and June 7th 2019, phase 2 between October 11th 2019 and December 11th 2019, and phase 3 between March 9th 2020 and July 16th 2020 .

\section{Phase 1}

Initially, stakeholders were asked to submit research questions pertaining to all aspects of major trauma via an online survey using a dedicated Twitter account (@delphi_natric). Engagement was raised by tagging leading individuals within the trauma community. Frequent tweet reminders were also published to continue raising engagement. Submitted questions were collated, anonymised, and then grouped into six distinct categories:
1. Brain injury
2. Rehabilitation
3. Trauma in older people
4. Pre-hospital care
5. Interventional care
6. Miscellaneous

Disagreements regarding question categorisation were resolved by consensus within steering subcommittees. Questions that did not clearly fit into categories 1-5 were categorised as miscellaneous. For each of the categories, a steering committee comprising subject matter experts and public representatives was formed. Duplicate questions were removed. Questions with a similar theme were altered by consensus and care was taken to ensure that the meaning of submitted questions was not altered.

\section{Phase 2}

Questions were prioritised by members of the trauma community by inviting stakeholders on Twitter to click on links to two surveys hosted on the SurveyMonkey platform. There were no restrictions on who could take part in the prioritisation process. The aim was to attract interest and contributions from all specialties that form part of the major trauma multi-disciplinary team in addition to patients and members of the public. Two surveys were designed, each containing three of the question categories. Survey one contained brain injury, rehabilitation, and elderly trauma. Survey two contained pre-hospital care, interventional care, and miscellaneous.
Respondents were asked to prioritise each question using a modified Likert scale (1-lowest research priority to 5highest research priority). The surveys remained open for 6 weeks, with 4 Tweets to act as an aide memoire to maximise stakeholder engagement. All results were anonymised, reviewed by the steering committees, and cut-offs were determined for inclusion in phase 3 without sight of the questions.

\section{Phase 3}

A final round of prioritisation was performed, and stakeholders were invited via Twitter to two separate surveys as per phase 2 . The surveys remained open for 4 months. Two email reminders were sent to all those who had engaged in phase 2, and 5 Tweets were publicised prior to closure of the survey. Results were anonymised and reviewed by the steering committee to agree on the final list of prioritised research questions.

During phases 2 and 3, only complete submissions within each subcategory were included in the analysis. For example, if a stakeholder ranked all questions within brain injury but did not complete the rest of the survey, only the brain injury responses were included in the final analysis.

The criteria for inclusion in the final list of prioritised questions varied between each of the six categories owing to different initial selected question numbers. The mean scores for each question were calculated and ranked.

\section{Results}

In total, 201 research questions were submitted by 65 stakeholders in phase 1. Once duplicated and similar questions were reviewed, amended, or removed by consensus, 186 questions were allocated to one of six categories and moved forward for prioritisation in Phase 2. In phase 1, 2 stakeholders were patients. The remainder were healthcare practitioners or researchers involved in major trauma. The number of questions taken forward within each category is summarised in Table 1.

Following review by the steering committees with consensus agreement on the criteria for inclusion, 56 questions were included in the final list of research priorities (Tables 2 , $3,4,5,6,7)$.

There was a significant variety in response numbers in phase 2, ranging from 37 responses in miscellaneous and interventional, to 66 in brain injury. In phase 3, all 64 stakeholders completed the surveys fully for the first 3 categories. This group consisted of 30 medical staff (46.9\%), 30 allied health professionals (46.9\%), and 4 full-time academics $(6.2 \%)$. Within each group, medical staffs were either surgical $(n=12)$, anaesthetic $(n=8)$, emergency department 
Table 1 Summary of questions included in each round (mean Likert cut-off score for inclusion in next round in parenthesis)

\begin{tabular}{llll}
\hline Category & $\begin{array}{l}\text { Questions } \\
\text { for phase 2 }\end{array}$ & $\begin{array}{l}\text { Questions } \\
\text { for phase 3 } 3\end{array}$ & $\begin{array}{l}\text { Prioritised } \\
\text { research ques- } \\
\text { tions }\end{array}$ \\
Total & 186 & 114 & 56 \\
\hline Brain injury & 29 & $17(3.22)$ & $10(3.41)$ \\
Rehabilitation & 47 & $25(3.20)$ & $10(3.65)$ \\
Trauma in older people & 21 & $15(3.43)$ & $10(3.42)$ \\
Pre-hospital & 26 & $23(3.35)$ & $10(3.67)$ \\
Interventional & 37 & $19(3.20)$ & $10(3.50)$ \\
Miscellaneous & 26 & $15(3.35)$ & $6(3.61)$ \\
\hline
\end{tabular}

$(n=6)$, or elderly care physicians $(n=4)$. Within the allied health professional groups, the majority were paramedics $(n=16)$. Sixty stakeholders completed the surveys fully for the last 3 categories. No patients or members of the public completed the surveys in phase 3 . All questions that were not included in the final list of research priorities are included in appendix 1.

The minimum mean score for inclusion after prioritisation was 3.41 to a maximum mean of 3.67 (Table 1). The final prioritised questions all had a Likert score of 1-2 by $<12 \%$ of respondents reflecting consensus that these were important research priorities.

\section{Brain injury}

In phase 2, 66 stakeholders took part in prioritisation and 64 in phase 3. Following review, 10 questions were included in the final list of research priorities.

\section{Rehabilitation}

In phase 2, 60 stakeholders contributed and 64 in phase 3.10 questions were included in the final list of research priorities.

\section{Trauma in older people}

In phase 2, 58 stakeholders took part and 64 in phase 3.10 questions were included in the final list of research priorities.

\section{Pre-hospital care}

In phase 2, 48 stakeholders took part and 60 in phase 3.10 questions were included in the final list of research priorities.

Table 2 Prioritised brain injury questions

How can we improve the provision of long-term care for patients with traumatic brain injury?

What is the optimal rehabilitation pathway for major trauma patients with mild traumatic brain injury?

What are the therapy needs of major trauma patients with mild traumatic brain injury?

What are the long-term needs of major trauma patients after traumatic brain injury?

Which patients derive benefit from cervical spine immobilisation?

When is it safe to restart anticoagulant therapy after traumatic brain injury?

What is the optimal mean arterial pressure for major trauma patients with a suspected head injury in the pre-hospital phase?

Which psychological interventions are the most effective for people with emotional difficulties as a result of traumatic brain injury?

When is it safe to restart antiplatelet therapy in patients with ischaemic heart disease after traumatic brain injury?

What are the predictors of a successful return to work after traumatic brain injury?

Table 3 Prioritised rehabilitation questions

Does early rehabilitation reduce morbidity and mortality in major trauma patients?

What are the most important factors that influence rehabilitation following major trauma?

Is there a regional variation in the provision of rehabilitation facilities for major trauma patients and does this impact upon clinical outcomes?

What is the best measure of quality of life for major trauma patients?

Which outcome measures should be used to evaluate the effectiveness of rehabilitation in major trauma patients?

What are the most effective psychological interventions for major trauma patients?

How can we prevent muscle wasting and maintain body mass in major trauma patients?

What is the prevalence of disability at six months, one and two years following index injury in major trauma patients?

What are the predictors of chronic pain following major trauma?

What are the predictors of a successful return to work following major trauma? 
Table 4 Prioritised elderly questions

What is the most effective method for triaging older major trauma patients?

Does caring for older major trauma patients in a dedicated major trauma ward improve clinical outcomes in major trauma centres?

Is there a difference in clinical outcomes in the delivery of care to older major trauma patients in a trauma unit versus major trauma centre?

Which criteria should be included in the triage tool for older major trauma patients following a fall from $<2 \mathrm{~m}$ ?

What is the ideal environment to care for older major trauma patients who have injuries to more than one body region?

Which cohort of older major trauma patients derive the greatest benefit from a comprehensive geriatric assessment?

Does frailty scoring in the emergency department improve clinical outcomes in older major trauma patients?

Should frailty be a triage criterion for older major trauma patients?

What are the clinical outcomes for older major trauma patients admitted with isolated rib fractures?

Is pre-hospital cervical immobilisation associated with poor outcomes for older major trauma patients?

Table 5 Prioritised pre-hospital care questions

Which pre-hospital trauma interventions have the greatest impact on clinical outcomes?

Do pre-hospital blood products improve outcomes in major trauma patients?

Which patient cohort derives the greatest benefit in morbidity and mortality from major trauma centre care?

Which physiological parameter is the best predictor of shock in major trauma?

How can the Major Trauma Triage Tool be adapted to effectively identify patients who need major trauma centre care?

What is the optimum blood product resuscitation fluid for major trauma patients?

Should direct oral anticoagulants (DOACs) be reversed in major trauma patients?

What are the most reliable markers of futility in the context of code red major trauma?

Is there an age-related difference in the clinical outcomes following redirection to a major trauma centre?

How should we reverse direct oral anticoagulants (DOACs) in major trauma patients?

Table 6 Prioritised interventional care questions

What is the optimal resuscitative approach to major trauma patients with concurrent traumatic brain injury and haemorrhage?

Is there a role for vasopressors in the hypotensive major trauma patient?

Can coagulation therapeutics or novel blood components provide better correction of trauma-induced coagulopathy than existing methods?

How can we reduce mortality from damage control surgery?

What is the pathophysiology of multiorgan failure in major trauma?

What is the optimal management for rib fractures in major trauma patients?

Is there a correlation between time to $\mathrm{CT}$ and survival in major trauma patients?

Is there a clinical benefit to early pre-emptive regional anaesthesia in blunt chest injury?

Do differing analgesic practices lead to variation in the outcomes for major trauma patients with blunt chest injury?

What are the mechanisms of cardiac dysfunction after trauma and what strategies, or interventions can protect the heart during resuscitation?

Table 7 Prioritised miscellaneous questions
What is the best measure of success for the delivery of care to major trauma patients?

What proportion of major trauma survivors return to work?

What inhibits some paramedics from bypassing to a major trauma centre?

How do we measure "success" in major trauma, is it mortality rates or quality of survival?

Is there an impact on patient care through the sharing or non-sharing of patient injuries and outcomes with pre-hospital providers?

What are the patient experiences of UK major trauma services? 


\section{Interventional}

In phase 2, 37 stakeholders took part and 60 in phase 3.10 questions were included in the final list of research priorities.

\section{Miscellaneous}

In phase 2, 37 stakeholders took part and 60 in phase 3.6 questions were included in the final list of research priorities.

\section{Overall top 10 priority questions across all categories}

The top 10 prioritised questions across all categories are as follows (Table 8):

\section{Discussion}

Our modified Delphi process has yielded a list of 56 highpriority research questions in the field of major trauma across six different subspecialty areas. This was achieved by utilising the collective expertise of patients, the public and the multi-disciplinary major trauma community. Across all 6 subcategories there is a clear emphasis on long-term patient-centred outcomes. This focus on long-term outcomes is prevalent across many recent Delphi studies, including those in bariatric [7] and hepatopancreatobiliary surgery [5].

NICE previously established 4 research priorities for trauma in their most recent management of major trauma guidelines. Our Delphi study has identified 1 duplicate research priority, namely the focus on trauma-induced coagulopathy and its prevention [3]. The remainder of the NICE priorities are largely interventional, reflecting their focus on initial clinical management. However, our study has identified a wider range of priorities, across the spectrum of major trauma, reflecting the multi-disciplinary approach to the management of major trauma.
Our Delphi study has focussed on UK priorities in the management of trauma. However, our identified research priorities are similar to those identified by similar methods recently in Australia and New Zealand, with a key focus on long-term patient-centred outcomes and the management of trauma in older people [16].

Responses were received from a mix of medical, academic, and allied health professional staff. Within the medical staff group, there was a range of specialties represented, including surgery, geriatric medicine, anaesthetics, and emergency medicine. The inclusion of non-interventional medical specialists such as those with expertise in looking after older patients was particularly welcomed. This ensured that non-surgical and non-interventional issues pertinent to the management of patients with major trauma were included. This is reflected in recent data, demonstrating that involvement of an elderly care multi-disciplinary team early in an elderly patient's major trauma journey significantly improves long-term outcomes [14].

This survey was principally publicised on Twitter, and therefore, members of the public were able to participate if they desired. Survey tweets were also 're-tweeted' by leading individuals in trauma surgery, and trauma societies in the UK to increase engagement. Furthermore, all steering committees included lay representation to ensure that patient perspective was included when prioritising questions. However, participation by members of the public was low, with only 2 patients submitting questions in phase 1 , and is therefore a limitation of our study findings. Future endeavours should focus upon patient engagement in priority setting for major trauma. One way to achieve this would be to host a patient focus group to seek the views of major trauma survivors to determine what mattered most to them when receiving major trauma care.

Phase 1 question submission remained open for 4 months to allow for adequate reflection on appropriate questions prior to submission. Phase 2 remained open for 6 weeks with strong engagement and participation noted. Phase 3 remained open for 4 months. The primary reason for the

Table 8 Top 10 prioritised questions across all categories

Which pre-hospital trauma interventions have the greatest impact on clinical outcomes?

Does early rehabilitation reduce morbidity and mortality in major trauma patients?

What is the optimal resuscitative approach to major trauma patients with concurrent traumatic brain injury and haemorrhage?

Do pre-hospital blood products improve outcomes in major trauma patients?

Is there a role for vasopressors in the hypotensive major trauma patient

Can coagulation therapeutics or novel blood components provide better correction of trauma-induced coagulopathy than existing methods

Which patient cohort derives the greatest benefit in morbidity and mortality from major trauma centre care?

How can we reduce mortality from damage control surgery?

What is the most effective method for triaging older major trauma patients?

Does caring for older major trauma patients in a dedicated major trauma ward improve clinical outcomes in major trauma centres? 
prolonged duration of phase 3 is attributed to the COVID19 pandemic. Phase 3 was opened immediately prior to UK lockdown restrictions being enforced. This may explain the increased number of responses across all 6 categories in phase 3 compared to phase 2 .

One major limitation we anticipated was that of survey fatigue. This is the tendency to not fully complete a survey when faced with several pages of questions, or reluctance to participate at all [15]. To mitigate this, we split the questions for phases 2 and 3 between 2 separate surveys released on 2 separate dates. Furthermore, only complete responses within each category were included to ensure that missing data did not adversely influence the prioritisation process.

A further limitation includes the increasing numbers of those prioritising questions from phase 2 to phase 3 . In phase 3, each subsection had a larger number of stakeholders prioritising questions than the same subsection in phase 2 as detailed in the results section. This means that some stakeholders only participated for one phase of the process, and therefore, there was limited continuity amongst stakeholders. This is permitted within a Delphi process. However, stakeholders that participated in all three rounds including question submission may be more likely to rank questions that they initially submitted more highly than others, artificially inflating the priority of those questions. In addition, we noted that the increasing numbers of those ranking in the 3rd phase also widened the demographic of the stakeholders, increasing the diversity of those participating to be more representative of the wider trauma community.

The final questions will be disseminated to funding bodies, and individual members of the trauma community will be encouraged to pursue these research questions with a multi-centre, multi-disciplinary patient-centred approach.

\section{Conclusion}

In summary, our modified Delphi process has resulted in a list of 56 questions that have been deemed by consensus amongst UK healthcare professionals delivering major trauma care to have the highest research priority in the field of the management of major trauma in the UK. Across all research categories, there was a clear emphasis on researching long-term patient outcomes, and how we can improve these through the entire trauma experience, from pre-hospital care, intervention in trauma, and rehabilitation. Furthermore, the establishment of key research priorities within trauma affecting older people reflects the changing demographic of major trauma within the UK. Future research projects and funding should seek to address these prioritised questions and also specifically target obtaining the views of major trauma patients. The findings of our study do lend themselves to being answered in a multi-centre multi-disciplinary approach to major trauma care.

\section{Appendix 1 Submitted questions from each category not included in final list of research priorities.}

\section{Brain injury}

Do patients on anticoagulants require CT head following head injury if there is no neurological compromise?

What are the mechanisms of brain injury associated coagulopathies, and how can they be diagnosed and treated?

Would a multi-centre audit of the management of patients with traumatic brain injury lead to improved clinical outcomes?

Does neuropsychiatry input for patients with traumatic brain injury improve clinical outcomes?

What effect do cognitive-based therapies have on functional outcomes for major trauma patients with post traumatic amnesia?

What predicts employment outcomes after traumatic brain injury?

Is paramedic sedation of patients with suspected traumatic brain injury associated with improved outcomes?

\section{Rehabilitation}

What is the interaction between comorbidities and major trauma in regards to survival and quality of survival?

How can we improve the patient experience of the major trauma pathway from admission to discharge?

Should major trauma rehabilitation be provided in an acute hospital, rehabilitation hospital or at home?

What are the current barriers to early supported discharge in major trauma patients?

How can the psychosocial support of major trauma patients be improved?

What is the optimal way to meet the needs of major trauma patients once discharged back to the community?

What are the most important psychological factors that influence rehabilitation following major trauma?

What is the socioeconomic impact of major trauma to patients, and how long does it last?

What is the incidence of depression amongst major trauma patients at six months, one year and two years following index injury?

What factors influence patient compliance during major trauma rehabilitation?

How can we use technology to assist with acute trauma rehabilitation?

How should we measure "major trauma", is it the injuries sustained or the on-going effect on patients life? 
How can family or carers be incorporated in to the rehabilitation pathway for major trauma patients?

What effect does cognitive-based therapy have on functional outcomes for major trauma patients with post traumatic amnesia?

What is the prevalence of chronic pain six months following index injury in major trauma patients?

\section{Trauma in older people}

Does frailty correlate with higher ISS in falls from $<2 \mathrm{~m}$ in the older major trauma population?

Should pre-hospital frailty screening be included in the pre-hospital major trauma triage tool?

Does pre-hospital cervical spine immobilisation improve clinical outcomes for older major trauma patients?

When should we CT the chest in older major trauma patients?

What is the incidence of traumatic brain injury in older major trauma patients and from what mechanisms?

\section{Interventional}

Are there any known molecular targets that can improve the clinical outcomes associated with multiorgan failure?

How do we improve analgesia for paediatric major trauma patients?

What are the barriers and facilitators to the use of regional anaesthesia in the ED with blunt chest wall injury for major trauma patients?

Should patients be allowed to self-mobilise in cases of suspected spinal injury?

Is resuscitative thoracotomy indicated in blunt traumatic cardiac arrest?

In a haemodynamically stable major trauma patient, what factors influence the optimal management strategy of pneumothorax/hemothorax?

Is length of stay and mortality affected by the time taken from decision to perform damage control surgery to knife to skin?

Can major trauma patients be kept pain free with single agent ketamine and single parameter EtCO2 monitoring for extended periods?

What is the optimal management for splenic lacerations in major trauma patients - do we need to routinely repeat imaging?

\section{Pre-hospital care}

What is the best way for pre-hospital personnel to identify major trauma in children?

Does involvement of PHEM sub-specialists in the pre-hospital environment improve clinical outcomes in major trauma patients?

What is the optimal analgesic regime for paediatric major trauma patients in the pre-hospital environment?

Should chest compressions be withheld in blunt traumatic cardiac arrest?

Do pre-hospital point-of-care devices improve clinical outcomes in major trauma patients?

How can we improve the rate of pre-hospital TXA administration to major trauma patients?

Is there a correlation between vehicle extrication times and clinical outcomes in major trauma patients?

What is the best method of maintaining normothermia in the prehospital environment?

Does early administration of IV calcium chloride decrease mortality in major trauma patients requiring blood products?

Does active or passive warming in the pre-hospital environment improve clinical outcomes in major trauma patients?

Does intra-nasal fentanyl improve the speed of onset and effectiveness of analgesia in the pre-hospital environment?

What are the common injury patterns associated with falls $<2 \mathrm{~m}$ ?

Does video link to a major trauma scene improve the clinical outcomes for major trauma patients?

\section{Miscellaneous}

What is the effect of trauma reconfiguration on the proportion of rural major trauma patients accessing major trauma centre care?

Should hot debriefing be a part of standard practice after code red major trauma calls?

What is the effect of trauma reconfiguration on 30 day mortality for major trauma patients?

What is the health economic impact of trauma reconfiguration?

What is the effect of trauma reconfiguration on the proportion of major trauma patients taken directly to major trauma centres?

What is the optimal mode of immobilisation (scoop/trauma board) for major trauma patients who require whole-body CT?

Should hot debriefing be a part of standard practice after major trauma calls?

Is there a saturation point of patient benefit from number of clinicians present at a trauma call?

What industries provide the greatest major trauma burden and how could health and safety in these industries be improved?

Funding Not applicable.

Availability of data and materials The datasets generated during and/or analysed during the current study are available from the corresponding author on reasonable request. 
Code availability Not applicable.

\section{Declarations}

Conflict of interest All authors declare they have no conflict of interest.

\section{References}

1. Murray CJ, Vos T, Lozano R, Naghavi M, Flaxman AD, Michaud C, et al. Disability-adjusted life years (DALYs) for 291 diseases and injuries in 21 regions, 1990-2010: a systematic analysis for the Global Burden of Disease Study 2010. Lancet. 2012;380(9859):2197-223.

2. Moran CG, Lecky F, Bouamra O, Lawrence T, Edwards A, Woodford $\mathrm{M}$, et al. Changing the system-major trauma patients and their outcomes in the NHS (England) 2008-17. EClinicalMedicine. 2018;2-3:13-21.

3. Major trauma: assessment and initial management. https://www. nice.org.uk/guidance/ng39/chapter/Recommendations-for-resea rch

4. Major trauma in older people: TARN: trauma audit research network. 2017. https://www.tarn.ac.uk/content/downloads/3793/ Major\%20Trauma\%20in\%20Older\%20People\%202017.pdf.

5. Knight SR, Pathak S, Christie A, Jones L, Rees J, Davies H, et al. Use of a modified Delphi approach to develop research priorities in HPB surgery across the United Kingdom. HPB (Oxford). 2019;21(11):1446-52.

6. Wilson MS, Blencowe NS, Boyle C, Knight SR, Petty R, Vohra RS, et al. A modified Delphi process to establish future research priorities in malignant oesophagogastric surgery. Surgeon. 2019;18:321-6.
7. Wilson MSJ, Knight S, Vaughan-Shaw P, Blakemore AI, O'Kane M, Boyle C, et al. A modified AUGIS Delphi process to establish research priorities in bariatric and metabolic surgery. Clin Obes. 2020;10(1):12344.

8. Wilson MSJ, Vaughan-Shaw P, Boyle C, Yong GL, Oglesby S, Skipworth R, et al. A modified AUGIS Delphi process to establish future research priorities in benign upper gastrointestinal surgery. World J Surg. 2020;44(4):1216-22.

9. NaTRIC-Centre For Trauma Sciences. https://www.c4ts.qmul. ac.uk/natric/natric.

10. Tiernan J, Cook A, Geh I, George B, Magill L, Northover J, et al. Use of a modified Delphi approach to develop research priorities for the association of coloproctology of Great Britain and Ireland. Colorectal Dis. 2014;16(12):965-70.

11. Burt CG, Cima RR, Koltun WA, Littlejohn CE, Ricciardi R, Temple LK, et al. Developing a research agenda for the American Society of Colon and Rectal Surgeons: results of a Delphi approach. Dis Colon Rectum. 2009;52(5):898-905.

12. Allan M, Mahawar K, Blackwell S, Catena F, Chand M, Dames N, et al. COVID-19 research priorities in surgery (PRODUCE study): a modified Delphi process. Br J Surg. 2020;107:e538-40.

13. NHS health research authority decision making tool. http://www. hra-decisiontools.org.uk/research/.

14. Duran SF, Mazzurco L, Palmer RM. Trauma consults by geriatricians: looking into the black box. Gerontol Geriatr Med. 2018;4:2333721418817668

15. O'Reilly-Shah VN. Factors influencing healthcare provider respondent fatigue answering a globally administered in-app survey. PeerJ. 2017;5:e3785.

16. Curtis K, Nahidi S, Gabbe B, Vallmuur K, Martin K, Shaban $\mathrm{R}$, Christey G. Identifying the priority challenges in trauma care delivery for Australian and New Zealand trauma clinicians. Injury. 2020;51(9):2053-8. 